\title{
Reliability, validity and responsiveness of the German self-reported foot and ankle score (SEFAS) in patients with foot or ankle surgery
}

\author{
Dariusch Arbab ${ }^{1 *}$ D, Katharina Kuhlmann², Christoph Schnurr ${ }^{3}$, Bertil Bouillon ${ }^{4}$, Christian Lüring ${ }^{5}$ and
} Dietmar König ${ }^{6}$

\begin{abstract}
Background: Patient-reported outcome measures are a critical tool in evaluating the efficacy of orthopedic procedures and are increasingly used in clinical trials to assess outcomes of health care. The intention of this study was to develop and culturally adapt a German version of the Self-reported Foot and Ankle Score (SEFAS) and to evaluate reliability, validity and responsiveness.

Methods: According to Cross Cultural Adaptation of Self-Reported Measure guidelines forward and backward translation has been performed. The German SEFAS was investigated in 177 consecutive patients. 177 Patients completed the German SEFAS, Foot and Ankle Outcome Score (FAOS), Short-Form 36 and numeric scales for pain and disability (NRS) before and 118 patients 6 months after foot or ankle surgery. Test-Retest reliability, internal consistency, floor and ceiling effects, construct validity and minimal important change were analyzed.

Results: The German SEFAS demonstrated excellent test-retest reliability with ICC values of 0.97. Cronbach's alpha (a) value of 0.89 demonstrated strong internal consistency. No floor or ceiling effects were observed for the German version of the SEFAS. As hypothesized SEFAS correlated strongly with FAOS and SF-36 domains. It showed moderate $(E S / S R M>0.5)$ responsiveness between preoperative assessment and postoperative follow-up.
\end{abstract}

Conclusion: The German version of the SEFAS demonstrated good psychometric properties. It proofed to be a valid and reliable instrument for use in foot and ankle patients.

Trial registration: DRKS00007585.

Keywords: SEFAS, Patient reported outcome measures, Questionnaire, Translation, Validation

\section{Background}

Patient reported outcome measurements (PROMS) can provide reliable and valid measures of patient's degree of pain, impairment, disability, and quality of life. They are a critical tool in evaluating the efficacy of orthopaedic procedures and are increasingly used in clinical trials to assess outcomes of health care $[1,2]$. The Self-reported Foot and Ankle Score (SEFAS) is a PROM for surgery of the foot and ankle, initially assessed in patients undergoing total ankle replacement due

\footnotetext{
* Correspondence: darbab@gmx.de

${ }^{1}$ Department of Orthopedic Surgery, Klinikum Dortmund, Member Faculty of Health Witten/Herdecke University, Beurhausstraße 40, 44137 Dortmund, Germany

Full list of author information is available at the end of the article
}

to osteoarthritis or inflammatory arthritis [3]. Further evaluation provided evidence in support of the reliability and validity using data from a large sample of patients undergoing surgery of a wide range of forefoot, hindfoot and ankle disorders [4]. In a systematic literature review about PROM's in hallux valgus the SEFAS showed good psychometric properties with good availability and less items compared to the Manchester-Oxford Foot Questionnaire [5]. The SEFAS is based on the New Zealand total ankle questionnaire [6], and was adapted by Cöster et al. in 2012 [3]. It contains 12 items, with 5 response options. The questionnaire covers different constructs, which are not reported separately in subscales. Pain, limitation of function and other symptoms are the 
main constructs [3]. Currently it has not been translated and evaluated in other languages.

The aim of this study was to translate the SEFAS into German language, culturally adapt it according to international guidelines, and to evaluate reliability, validity and responsiveness.

\section{Methods}

The study was approved by Ethics Commission of the Faculty of Medicine of Cologne University (ref 15-252, DRKS-ID DRKS00007585, IRB00003528 Klinikum der Universitat zu Koln IRB \#1, 2014-04-28) and performed in accordance with the Declaration of Helsinki. Written informed consent from all participants was obtained.

\section{Translation}

Forward and backward translation of the SEFAS was performed according to international guidelines of the International Society for Pharmacoeconomics and Outcomes Research (ISPOR) [7]. Two bilingual translators with different profiles (one physician) whose native language was German independently translated the English version forward into German. Two native English speakers without medical education performed backward translation of the provisional German questionnaire into English. Translators and a group of foot and ankle surgeons consolidated all versions of the questionnaire and developed a final version for field testing. The final version was tested on 15 patients with foot or ankle disorders to ascertain acceptance and comprehension (Additional files 1 and 2).

\section{Patients and validation procedure}

From November 2014 to January 2016 a total of 177 patients undergoing surgery of the foot or ankle were consecutively recruited at a single institution. Eligibility criteria included adult patients undergoing primary foot or/and ankle surgery for osteoarthritis, deformity, rheumatoid arthritis, impingement of the ankle, tendon disorders or bone defect. Patients were asked to complete the German SEFAS, the German Foot and Ankle Outcome Score (FAOS) the German Short-Form 36 Health Survey (SF-36) and a numeric scale for pain and disability (NRS). SEFAS, FAOS, SF-36 and NRS were completed 3-14 days before surgery ( $\mathrm{t} 1$ ) and again on the morning before surgery ( $\mathrm{t} 2$ ) for reliability testing. 6 months after surgery (t3) all participants were asked to complete SEFAS a last time.

\section{Instruments}

The SEFAS is a 12-item questionnaire covering different constructs, which are not separately reported in subscales. We defined 3 subscales consisting of: pain (p) (seven items, question 1, 2, 5, 8, 9, 11, 12), (limitation of) function (lof) (three items question $3,6,7$ ) and others (o) (2 items question $4,10)$. Patients score each question on a five-point Likert scale scored from 0 to 4 , with 0 representing the worst stage and the sum of 48 representing normal function [3].

The FAOS is a 42-item instrument to evaluate symptoms and functional limitations related to the foot and ankle [8]. It consists of five domains: pain (p), other symptoms (s), activities of daily living (adl), sport and recreational activities $(\mathrm{s} / \mathrm{r})$, and foot-and ankle-related quality of life (qol). Each item is scored on a five-point Likert scale from 0 to 4 , with 4 representing the worst stage. The total score is transformed to a scale from 0 to 100, like SEFAS low numbers represent more severe stages. FAOS has been translated into German and validated as a reliable instrument for use in foot and ankle patients [9].

The SF-36 instrument is a widely used generic patientreported instrument to measure health related quality of life. It consists of eight domains: physical functioning (pf), role physical (rp), role emotional (re), social functioning (sf), mental health $(\mathrm{mh})$, energy/vitality $(\mathrm{e} / \mathrm{v})$, pain $(\mathrm{p})$, general health perception (gh). It has been translated and validated into German [10].

The NRS were used to determine pain and disability of the foot and ankle. On a $0-10$ scale 10 represents the most severe pain or disability.

\section{Statistical analysis}

The SEFAS and FAOS subscale scores were entered into a Microsoft Excel spreadsheet (Microsoft Corporation, Redmond WA) and analyzed using SPSS v24 (SPSS Inc. Chicago, Illinois). A $p$-value $<0.05$ was considered to indicate statistical significance.

\section{Reliability}

\section{Reproducibility}

Reproducibility as test-retest reliability was assessed by calculating interclass correlation coefficient (ICC, Two-way Random Effect Model Absolute Agreement Definition) between SEFAS completed at the first visit 3-14 days before surgery (step 1) and second time before surgery (step 2). An ICC value of 0.7 and above was considered as good [11, 12].

\section{Internal consistency}

Reliability also includes internal consistency [12]. Internal consistency is the extent to which items within a scale are homogeneous, thus measuring the same construct $[13,14]$. Cronbach's alpha $(\alpha)$ coefficient was calculated to assess internal consistency of the SEFAS items. Values of alpha of 0.7, 0.8 and 0.9 are considered to represent fair, good and excellent degree of internal consistency, respectively [15].

\section{Floor and ceiling effects}

Floor and ceiling effects were considered to exist if more than $15 \%$ of responses reached lowest or highest possible score. A high floor or ceiling effect could make it difficult to measure changes after intervention like surgery $[3,16,17]$. 


\section{Validity}

\section{Construct validity}

Describes the extent to which a score relates to other scores [17]. As no gold standard exists the SEFAS and its defined subscales were compared to FOAS and SF-36 and NRS pain and disability using non-parameteric correlation coefficients (Spearman's Rho). Correlation coefficients $<0.4$ were considered as low, 0.4-0.59 as moderate and 0.6-0.79 as high correlation [18]. For convergent validity Cöster et al. 2014 expected high correlation between SEFAS and the FAOS domains p, adl, qol and with the SF-36 domains pf and bp [4]. We hypothesized for the SEFAS $\mathrm{p}$ a high correlation with FAOS domain p, adl and SF-36 domain bp, pf and NRS. SEFAS lof and the FAOS dimension sr,adl, qol and SF-36 domain pf should show high correlations as well. For discriminant validity low to moderate correlations were expected between SEFAS and SF-36 domain gh, re and mh [4].

\section{Responsiveness}

Responsiveness is the extent to which a questionnaire is able to detect changes over time or due to an intervention such as surgery [19]. All patients completed SEFAS before surgery (t1) and 6 months after surgery (t3). To test responsiveness effect size (ES) and standardized response means (SRM) were calculated. ES is calculated as the difference between the means before and after intervention divided by the standard deviation (SD) of the same measure before treatment [12]. SRM is calculated as the difference between the means before and after treatment divided by the SD of the change. For both, ES and SRM, values of 0.2, 0.5 and 0.8 were regarded as small, moderate and large effects, respectively [12].

\section{Minimal important change (MIC) (laut Dawson ist hier "minimal important change" der richtige Ausdruck - Siehe meine letzte mail)}

Minimal important change (MIC) is the smallest change in a treatment outcome that a patient or physician would identify as important. MIC describes a threshold above which outcome is experienced as relevant by the patient and avoids the problem of bare statistical significance [20]. One distribution-based approach to calculate MIC is the minimal detectable change (MDC). It is defined as minimum amount of change that can be considered above the threshold of a measurement error. If the change in a score is higher than MDC, it can be considered as a true change [21]. It is calculated from the standard error of measurement (SEM), which is related to the internal consistency/reliability of the score (Cronbach's alpha). (SEM = Standard deviation $* \sqrt{1}$ - Cronbach's alpha). To allow comparisons with other studies, the MDC was calculated based on the confidence level of 90\% (MDC90: MDC $=1,65 *$ SEM * $\sqrt{2}$ ) [22]. In order to estimate significant change of the scores over time, we performed likelihood ratio tests after applying mixed-effects linear regression with random patient effects to account for repeated (longitudinal) measurements on the same patient $[23,24]$.

\section{Results}

One hundred seventy seven patients, 130 women and 47 men, with a mean age of 57 years (18-92) undergoing surgery of the foot or ankle were consecutively recruited at a single institution and completed the baseline 3-14 days before surgery (t1). On the morning before surgery (t2) 145 patients completed MOXFQ to determine reliability. 6 months after surgery (t3) 117 patients completed MOXFQ, FAOS, SF-36 and NRS to test responsiveness. 118 patients were undergoing forefoot, 56 patients hindfoot or ankle surgery.

\section{Reliability \\ Reproducibility}

The SEFAS demonstrated excellent test-retest reliability with ICC values of 0.95 for limitation of function, 0.97 for pain, 0.96 other symptoms and 0.97 for the SEFAS total. The mean indexes for the baseline and the reliability assessments were 48.4 (Standard deviation (SD) 19.7) and 49.7 (SD 20.7), respectively (Table 1 ).

\section{Internal consistency}

Cronbach's alpha $(\alpha)$ value of 0.89 for SEFAS total and 0.87 for pain demonstrated strong internal consistency. Lof values of 0.79 still showed fair internal consistency, whereas os demonstrated low consistency (0.35) (Table 1).

\section{Floor and ceiling effects}

No floor or ceiling effects were observed for the German version of the SEFAS (Table 2).

Table 1 Reliability - Cronbach's alpha and ICC

\begin{tabular}{llllllll}
\hline & & \multicolumn{2}{l}{ Reliabilität } & & & Consistency \\
\cline { 3 - 6 } \cline { 3 - 6 } & & Test Mean t1 (SD) & Retest Mean t2 (SD) & Cronbach's a t1 & Cronbach's a t2 & ICC & $0.973(0.960 ; 0.981)$ \\
\hline SEFAS & pain & $52.05(19.29)$ & $52.69(19.33)$ & 0.858 & 0.870 & $0.954(0.934 ; 0.968)$ \\
& Iof & $47.13(23.93)$ & $48.54(24.90)$ & 0.747 & 0.790 & $0.959(0.941 ; 0.972)$ \\
& OS & $45.92(27.57)$ & $47.49(29.48)$ & 0.223 & 0.354 & $0.970(0.957 ; 0.979)$ \\
\hline
\end{tabular}

Reliability with test-retest reliability and internal consistency of the German SEFAS

lof $=$ limitation of function, $\mathrm{o}=$ others, ICC $=$ interclass correlation coefficient, $\mathrm{SD}=$ standard deviation, $\mathrm{t} 1=$ first assessment, $\mathrm{t} 2=$ second assessment,

$\mathrm{Cl}=$ confidence interval 
Table 2 Floor and ceiling effects

\begin{tabular}{|c|c|c|c|c|c|c|c|c|}
\hline \multirow[t]{2}{*}{ SEFAS } & \multicolumn{4}{|l|}{ t1 } & \multicolumn{4}{|l|}{ t2 } \\
\hline & pain & lof & os & total & pain & lof & OS & total \\
\hline $\min (0)$ & $0.7 \%$ & $4.1 \%$ & $9.0 \%$ & $0.7 \%$ & $0.7 \%$ & $4.1 \%$ & $8.2 \%$ & $0 \%$ \\
\hline $\max (100)$ & $0 \%$ & $0 \%$ & $3.5 \%$ & $0 \%$ & $0 \%$ & $0 \%$ & $4.8 \%$ & $0 \%$ \\
\hline all $(\min +\max )$ & $0.7 \%$ & $4.1 \%$ & $12.5 \%$ & $0.7 \%$ & $0.7 \%$ & $4.1 \%$ & $13 \%$ & $0 \%$ \\
\hline
\end{tabular}

Floor and ceiling effects are considered to be present when more than $15 \%$ of the individuals reach the highest or lowest possible numeric value of a score

$\mathrm{t} 1$ = 3-14 days before surgery, $\mathrm{t} 2=$ morning before surgery, lof = limitation of function, os = others

\section{Validity}

\section{Construct validity}

To examine construct validity the Spearman's correlation coefficients between SEFAS, SF-36, FAOS and NRS were examined and shown in Table 3. We hypothesized that SEFAS subscale os will show low correlation to any of the FAOS domains. Convergent validity of the SEFAS was shown with strong correlations $(0.6-0.79)$ with the FAOS and its subscales and SF-36 domains pf, gph and bp. As hypothesized SEFAS p subscale correlated strongly with FAOS domain p, adl, and SF-36 domains bp and pf. SEFAS lof and FAOS sr, adl, qol and SF-36 domain pf showed high correlation as expected. Moderate correlation could be calculated for SEFAS and NRS, whereas SEFAS $p$ and NRS showed high construct validity. Discriminant validity with low to moderate correlation could be calculated for SF-36 gh, re, mh and gmh. All these findings were statistically significant $(p<0.05)$.

\section{Responsiveness}

Table 4 shows the responsiveness of the SEFAS. With an effect size of 0.71 the SEFAS demonstrated moderate (ES/ SRM > 0.5) responsiveness between preoperative assessment (t2) and postoperative follow-up (t3) indicating that a good degree of change was detected following surgery. The highest effect size showed the pain subscale (1.06).

The SEM was 2.39, 2.13, 3.01 and 2.16 for the German SEFAS lof, p, os and total, respectively. MDC90 (90\% confidence level) were 5.58, 4.97, 7.03 and 5.04 for domains lof, p, os and total, respectively. The mean difference (15.04) between preoperative and postoperative assessment is shown on Table 4. The German SEFAS showed higher changes than the MDC (5.04) which indicates true changes [19].

\section{Discussion}

Limited internationally used self-reported instruments are available to assess outcome of foot and ankle surgery. There is a need for reliable, valid, free and in native languages available reported outcome measures used for clinical trials and health care evaluation. The SEFAS has been initially validated for patients with ankle osteoarthritis and later for patients with a wide variety of different disorders of the foot and ankle proofing good validity, reliability and responsiveness [3, 4]. It is available in English and Swedish and has not been culturally adapted and translated in any other language.

In this study the English version of the SEFAS was crossculturally adapted and translated to German according to

Table 3 Validity

\begin{tabular}{|c|c|c|c|c|c|}
\hline \multirow[t]{3}{*}{$N=177$} & & \multicolumn{4}{|l|}{ SEFAS } \\
\hline & & \multirow{2}{*}{$\begin{array}{l}\text { pain } \\
\text { Spearman }\end{array}$} & \multirow{2}{*}{$\begin{array}{l}\text { limitation of function } \\
\text { Spearman }\end{array}$} & \multirow{2}{*}{$\begin{array}{l}\text { other symptoms } \\
\text { Spearman }\end{array}$} & \multirow{2}{*}{$\begin{array}{l}\text { total } \\
\text { Spearman }\end{array}$} \\
\hline & & & & & \\
\hline \multirow[t]{6}{*}{ FAOS } & symptoms & 0.595 & 0.565 & 0.441 & 0.625 \\
\hline & pain & 0.792 & 0.748 & 0.444 & 0.765 \\
\hline & activities of daily living & 0.792 & 0.778 & 0.418 & 0.771 \\
\hline & sports/recreation & 0.739 & 0.725 & 0.371 & 0.712 \\
\hline & quality of life & 0.706 & 0.672 & 0.293 & 0.642 \\
\hline & total & 0.801 & 0.766 & 0.434 & 0.775 \\
\hline \multirow[t]{10}{*}{ SF36 } & physical functioning & 0.691 & 0.665 & 0.391 & 0.673 \\
\hline & role physical & 0.631 & 0.578 & 0.355 & 0.597 \\
\hline & bodily pain & 0.777 & 0.691 & 0.306 & 0.673 \\
\hline & general health & 0.470 & 0.398 & 0.272 & 0.433 \\
\hline & Energy/vitality & 0.377 & 0.373 & 0.145 & 0.332 \\
\hline & social functioning & 0.638 & 0.513 & 0.270 & 0.528 \\
\hline & role emotional & 0.470 & 0.477 & 0.298 & 0.479 \\
\hline & mental health & 0.379 & 0.347 & 0.099 & 0.301 \\
\hline & general physical health & 0.718 & 0.683 & 0.403 & 0.685 \\
\hline & general mental health & 0.416 & 0.389 & 0.181 & 0.358 \\
\hline NRS & total & 0.617 & 0.508 & 0.283 & 0.542 \\
\hline
\end{tabular}

Construct validity: Baseline data and assessment of Spearman's correlation coefficients and of SEFAS, FAOS, SF-36 and NRS 
Table 4 Responsiveness

\begin{tabular}{|c|c|c|c|c|c|c|c|c|}
\hline & & \multicolumn{5}{|l|}{ Responsiveness } & \multicolumn{2}{|l|}{ MID } \\
\hline & & Per-OP Mean (SD) & Post-OP Mean (SD) & Mean Difference (SD) & ES & SRM & MDC 90 & SEM \\
\hline \multirow[t]{4}{*}{ SEFAS } & pain & $52.06(19.25)$ & $29.37(23.47)$ & $22.69(22.82)$ & 1.06 & 0.99 & 4.97 & 213 \\
\hline & lof & $47.51(25.30)$ & $29.20(25.39)$ & $18.31(25.55)$ & 0.72 & 0.72 & 5.58 & 2.39 \\
\hline & os & $51.14(27.59)$ & $47.39(27.78)$ & $3.75(32.32)$ & 0.14 & 0.12 & 7.03 & 3.01 \\
\hline & total & $50.37(20.58)$ & $35.33(21.83)$ & $15.04(23.18)$ & 0.71 & 0.65 & 5.04 & 2.16 \\
\hline
\end{tabular}

Responsiveness of the SEFAS showing mean changes, effect size and minimal important difference demonstrating minimal detectable change and standard error of measurement

lof = limitation of function, $\mathrm{o}=$ others, $\mathrm{SD}=$ standard deviation, $\mathrm{ES}=$ effect size, $\mathrm{SRM}=$ standardized response means, $\mathrm{MID}=$ minimal important difference,

$\mathrm{MDC}=$ minimal detectable change, $\mathrm{SEM}=$ standard error of measurement

the official guidelines of the International Society for Pharmacoeconomics and Outcomes Research (ISPOR) [7]. Originally the questionnaire covers different constructs, which are not reported separately in subscales 3$]$. We defined the main constructs pain, limitation of function and others as subscales to enhance comparability to FAOS and SF-36 domains.

The psychometric analyses demonstrated that the German version of the SEFAS has good validity, reliability and responsiveness in patients undergoing foot and ankle surgery.

Test-retest reliability of the German SEFAS showed excellent results with ICC values ranging from 0.97 for the SEFAS and 0.95 for limitation of function, 0.97 for pain and 0.96 other symptoms. These results are comparable to the Swedish version [4]. The mean indexes for the baseline and the reliability assessments were 48.4 (Standard deviation (SD) 19.7) and 49.7 (SD 120.7), respectively.

Strong internal consistency has been demonstrated for the SEFAS (Cronbach's alpha 0.89) and SEFAS pain (0.87) of the German version. The limitation of function (0.79) subscale showed fair internal consistency, whereas the other symptoms subscale demonstrated low consistency (0.35). Low internal consistency for os subscale was expected due to inhomogeneous questions asking for different constructs. The Swedish SEFAS showed similar results (0.84) for internal consistency. No floor or ceiling effects were observed, which is in line with the Swedish version.

Construct validity was determined by comparing the German SEFAS with the German SF-36 and FAOS. The German SEFAS showed strong correlations $(>0,6)$ with FAOS and its subscales and SF-36 domains physical functioning, general physical health and bodily pain. As hypothesized SEFAS pain subscale correlated strongly with FAOS domains pain and activity of daily living, SF-36 domains bodily pain and physical functioning. SEFAS domain limitation of functioning showed strong correlation with FAOS sports/recreation, activities of daily living and quality of life and with SF-36 domain physical functioning. Cöster et al. 2014 could confirm $80 \%$ of their predefined hypotheses showing strong correlations between SEFAS and 4 of the 5 subscales of FAOS [4]. In our study the German SEFAS showed strong correlations with all FAOS subscales.
Discriminant validity with low to moderate correlation was found for the German SEFAS and SF-36 domains general health, role emotional, mental health and general physical health.

Table 4 illustrates the responsiveness of the German SEFAS which demonstrated moderate $(E S / S R M>0.5)$ responsiveness in 118 patients with an effect size of 0.71 between preoperative (t2) and postoperative follow-up (t3). Cöster et al. 2014 showed higher effect sizes $(E S>1)$ in their population (70 patients) [4].

Evaluation of the German SEFAS showed similar results to the Swedish SEFAS [4]. Cultural differences, smaller number of patients and different foot pathologies and surgeries may be the reason for differing results in some aspects. A definition of subscales was not intended by the developers of the questionnaire but allowed a more specific examination of psychometric properties.

A limitation of the study is the inhomogeneity of the patient sample regarding sex and age. We see this as a result of the included pathologies which concern mainly women and are not restricted in age of occurrence. For this reason, these heterogeneities can also be found in comparable studies.

Unfortunately it was not possible to evaluate reliability and responsiveness based on the total sample size of 177 patients due to a lower response rate in repeated assessments. However, a sample size of $>100$ patients could be achieved at all time which we consider sufficient and representative. Regarding the assessment of Minimal important change, in our study only distribution-based methods were used. These are not as patient-centered as anchorbased methods and do not include a clinical criterion which limits their interpretability in a clinical setting.

\section{Conclusions}

In conclusion the German translation of the SEFAS demonstrated good psychometric properties. Our study demonstrated that the German questionnaire is a valid and reliable instrument for patients with foot and ankle disorders and can be used as a tool for evaluating the efficacy of surgical procedures and in clinical trials to assess outcomes of health care. 


\section{Additional files}

Additional file 1: German Translation of SEFAS Questionnaire. (PDF 317 kb)

Additional file 2: English SEFAS Questionnaire. (PDF $32 \mathrm{~kb}$ )

\section{Abbreviations}

adl: subdomain activities of daily living; e/v: Subdomain energy/vitality; ES: Effect size (ES); FAOS: Foot and Ankle Outcome Score; gh: Subdomain general health perception; ICC: Interclass correlation coefficient; MDC: minimal detectable change; mh: Subdomain mental health; MIC: Minimal important change; NRS: Numeric scales for pain and disability; p: Subdomain pain; p: Subdomain pain (p); pf: Subdomain physical functioning; Qol: Subdomain quality of life (qol); re: Subdomain role emotional; rp: Subdomain role physical; s: Subdomain other symptoms; s/ r: Subdomain sport and recreational activities; SEFAS: Self-reported Foot and Ankle Score; SEM: standard error of measurement; sf: Subdomain;

SF36: Short-Form 36: SRM: Standardized response means

\section{Acknowledgements}

All individuals who contributed towards the article are already included in the authors list.

\section{Availability of data and materials}

The datasets analyzed during the current study are available from the corresponding author on reasonable request.

\section{Authors' contributions}

The authors were responsible for the study design (AD, KK, KD), data collection and analysis (AD, KK, SK, BB, LC), writing of the manuscript (AD, KK, $S K, K D)$, and performing substantial revisions of the manuscript (AD, KK, SK, $B B, L C, K D)$. All authors read and approved the final manuscript.

\section{Funding}

No funding

\section{Ethics approval and consent to participate}

The study was approved by Ethics Commission of the Faculty of Medicine of Cologne University (ref 15-252, DRKS-ID DRKS00007585, IRB00003528 Klinikum der Universitat zu Koln IRB \#1, 2014-04-28) and performed in accordance with the Declaration of Helsinki.

\section{Consent for publication}

Not applicable.

\section{Competing interests}

The authors declare that they have no competing interests.

\section{Publisher's Note}

Springer Nature remains neutral with regard to jurisdictional claims in published maps and institutional affiliations.

\footnotetext{
Author details

${ }^{1}$ Department of Orthopedic Surgery, Klinikum Dortmund, Member Faculty of Health Witten/Herdecke University, Beurhausstraße 40, 44137 Dortmund, Germany. ${ }^{2}$ Medical University Düsseldorf, Moorenstraße 5, 40225 Düsseldorf, Germany. ${ }^{3}$ Department of Orthopedic Surgery, St. Vinzenz Krankenhaus, Schlossstraße 85, 40447 Düsseldorf, Germany. ${ }^{4}$ Department of Traumatology and Orthopedic Surgery, Cologne-Merheim Medical Center (CMMC), University of Witten/ Herdecke, Ostmerheimer Str. 200, 51109 Cologne, Germany. ${ }^{5}$ Department of Orthopedic Surgery, Klinikum Dortmund, Beurhausstraße 40, 44137 Dortmund, Germany. ${ }^{6}$ LVR Clinics of Orthopedic Surgery, Horionstraße 2, 41479 Viersen, Germany.
}

Received: 23 May 2017 Accepted: 3 October 2017

Published online: 10 October 2017

\section{References}

1. Parker J, Nester CJ, Long AF, Barrie J. The problem with measuring patient perceptions of outcome with existing outcome measures in foot and ankle surgery. Foot Ankle Int. 2003;24:56-60.

2. Department of Health. Our NHS our future: NHS next stage review - interim report. London: Department of Health, 2007

3. Cöster M, Karlsson MK, Nilsson JÅ, Carlsson A. Validity, reliability, and responsiveness of a self-reported foot and ankle score (SEFAS). Acta Orthop. 2012:83(2):197-203.

4. Cöster MC, Bremander A, Rosengren BE, Magnusson H, Carlsson A, Karlsson MK. Validity, reliability, and responsiveness of the self-reported foot and ankle score (SEFAS) in forefoot, hindfoot, and ankle disorders. Acta Orthop. 2014;85(2):187-94.

5. Schrier JC, Palmen LN, Verheyen CC, Jansen J, Koëter S. Patient-reported outcome measures in hallux valgus surgery. A review of literature. Foot Ankle Surg. 2015:21(1):11-5.

6. Hosman AH, Mason RB, Hobbs T, Rothwell AGA. New Zealand national joint registry review of 202 total ankle replacements followed for up to 6 years. Acta Orthop. 2007;78(5):584-91.

7. Wild D, Grove A, Martin M, Eremenco S, Mcelroy S, VerjeeLorenz A, Erikson P. Principles of good practice for the translation and cultural adaptation process for patient-reported outcomes (prO) measures: report of the ISpOr task force for translation and cultural adaptation. Value Health. 2005;8:94-104.

8. Roos EM, Brandsson S, Karlsson J. Validation of the foot and ankle outcome score for ankle ligament reconstruction. Foot Ankle Int. 2001;22:788-94.

9. van Bergen CJ, Sierevelt IN, Hoogervorst P, Waizy H, van Dijk CN, Becher C. Translation and validation of the German version of the foot and ankle outcome score. Arch Orthop Trauma Surg. 2014;134(7):897-901.

10. Bullinger M. German translation and psychometric testing of the SF-36 health survey: preliminary results from the IQOLA project. International quality of life assessment. Soc Sci Med. 1995;41:1359-66.

11. Weir JP. Quantifying test-retest reliability using the intraclass correlation coefficient and the SEM. J Strength Cond Res. 2005;19(1):231-40.

12. Streiner D, Norman G. Health measurement scales: a practical guide to their development and use. New York: Oxford University Press; 2008.

13. Scholtes VA, Terwee CB, Poolman RW. What makes a measurement instrument valid and reliable? Injury. 2011;42:236-40.

14. Lohr KN, Aaronson NK, Alonso J, Burnam MA, Patrick DL, Perrin EB, Roberts JS. Evaluating quality-of-life and health status instruments: development of scientific review criteria. Clin Ther. 1996;18(5):979-92.

15. Bland JM, Altman DG. Cronbach's alpha. BMJ. 1997;22(314):572.

16. Cöster MC, Rosengren BE, Bremander A, Brudin L, Karlsson MK. Comparison of the self-reported foot and ankle score (SEFAS) and the American orthopedic foot and ankle society score (AOFAS). Foot Ankle Int. 2014;35(10):1031-6.

17. Terwee CB, Bot SD, de Boer MR, van der Windt DA, Knol DL, Dekker J. Quality criteria were proposed for measurement properties of health status questionnaires. J Clin Epidemiol. 2007:60(1):34-42.

18. Dawson J, Boller I, Doll H, Lavis G, Sharp R, Cooke P, Jenkinson C. The MOXFQ patient-reported questionnaire: assessment of data quality, reliability and validity in relation to foot and ankle surgery. Foot. 2011;21:92-102.

19. Wright JG, Young NLA. Comparison of different indices of responsiveness. J Clin Epidemiol. 1997:50:239-46.

20. Jaeschke R, Singer J, Guyatt GH. Measurement of health status. Ascertaining the minimal clinically important difference. Control Clin Trials. 1989:10(4):407-15.

21. Wright A, Hannon J, Hegedus EJ, Kavchak AE. Clinimetrics corner: a closer look at the minimal clinically important difference (MCID). J Man Manipulative Ther. 2012;20:160-6.

22. McHorney CA, Tarlov AR. Individual-patient monitoring in clinical practice: are available health status surveys adequate? Qual Life Res. 1995;4:293-307.

23. Dawson J, Boller I, Doll H, Lavis G, Sharp R, Cooke P, Jenkinson C. Minimally important change was estimated for the Manchester-Oxford foot questionnaire after foot/ankle surgery. J Clin Epidemiol. 2014;67(6):697-705.

24. Garcés JB, Winson I, Goldhahn S, Castro MD, Swords MP, Grujic L, Rammelt S, Sands AK. Reliability, validity and responsiveness of the Spanish Manchester-Oxford foot questionnaire (SEFAS) in patients with foot or ankle surgery. Foot Ankle Surg. 2016;22:59-70 